\title{
High Order Numerical Methods for the Investigation of the Two Dimensional Richtmyer- Meshkov Instability
}

\author{
W.-S. Don, D. Gottlieb, C.-W. Shu, L. Jameson
}

\section{November 26, 2001}

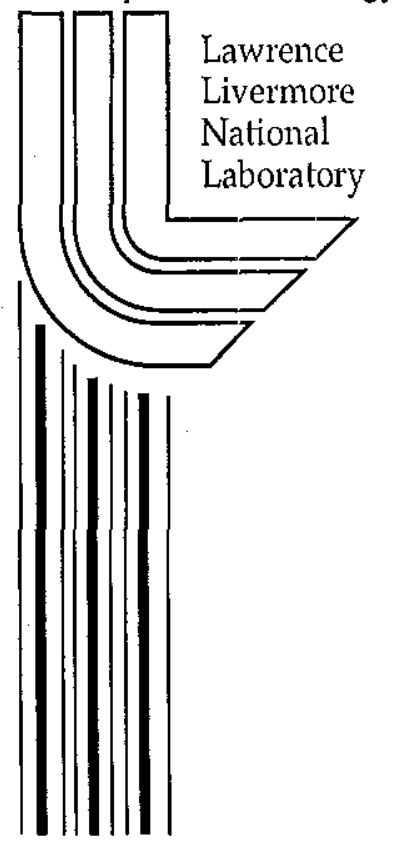




\section{DISCLAIMER}

This document was prepared as an account of work sponsored by an agency of the United States Government. Neither the United States Government nor the University of California nor any of their employees, makes any warranty, express or implied, or assumes any legal liability or responsibility for the accuracy, completeness, or usefulness of any information, apparatus, product, or process disclosed, or represents that its use would not infringe privately owned rights. Reference herein to any specific commercial product, process, or service by trade name, trademark, manufacturer, or otherwise, does not necessarily constitute or imply its endorsement, recommendation, or favoring by the United States Government or the University of California. The views and opinions of authors expressed herein do not necessarily state or reflect those of the United States Government or the University of California, and shall not be used for advertising or product endorsement purposes.

This work was performed under the auspices of the U. S. Department of Energy by the University of California, Lawrence Livermore National Laboratory under Contract No. W-7405-Eng-48.

This report has been reproduced directly from the best available copy.

Available electronically at http://www.doc.gov/bridge

Available for a processing fee to U.S. Department of Energy

And its contractors in paper from

U.S. Department of Energy

Office of Scientific and Technical Information

P.O. Box 62

Oak Ridge, TN 37831-0062

Telephone: (865) 576-8401

Facsimile: (865) 576-5728

E-mail: reports@adonis.osti.gov

Available for the sale to the public from

U.S. Department of Commerce

National Technical Information Service

5285 Port Royal Road

Springfield, VA 22161

Telephone: (800) 553-6847

Facsimile: (703) 605-6900

E-mail: orders@ntis.fedworld.gov

Online ordering: http://www.ntis.gov/ordering.htm

OR

Lawrence Livermore National Laboratory

Technical Information Department's Digital Library

http://www.llnl.gov/tid/Library.html 


\title{
High Order Numerical Methods for the Investigation of the Two Dimensional Richtmyer-Meshkov Instability
}

\author{
Wai-Sun Don, David Gottlieb, Chi-Wang Shu \\ Division of $\Lambda$ pplied Mathematics, Brown University \\ Leland Jameson \\ Lawrence Livermore National Laboratory
}

\begin{abstract}
For flows that contain significant structure, high order schemes offer large advantages over low order schemes. Fundamentally, the reason comes from the truncation error of the differencing operators. If one examines carefully the expression for the truncation error, one will see that for a fixed computational cost that the error can be made much smaller by increasing the numerical order than by increasing the number of grid points. One can readily derive the following expression which holds for systems dominated by hyperbolic effects and advanced explicitly in time,

$$
\text { flops }=\text { const } * p^{2} * \frac{k^{(d+1)(p+1) / p}}{E^{(d+1) / p}}
$$

where flops denotes floating point operations, $p$ denotes numerical order, $d$ denotes spatial dimension, where $E$ denotes the truncation error of the difference operator, and where $k$ denotes the Fourier wavenumber. For flows that contain structure, such as turbulent flows or any calculation where, say, vortices are present, there will be significant energy in the high values of $k$. Thus, one can see that the rate of growth of the flops is very different for different values of $p$. Further, the constant in front of the expression is also very different. With a low order scheme, one quickly reaches the limit of the computer. With the high order scheme, one can obtain far more modes before the limit of the computer is reached. Here we examine the application of spectral methods and the Weighted Essentially Non-Oscillatory (WENO) scheme to the Richtmyer-Meshkov Instability. We show the intricate structure that these high order schemes can calculate and we show that the two methods, though very different, converge to the same numerical solution indicating that the numerical solution is very likely physically correct.
\end{abstract}




\section{High Order Numerical Methods for the Investigation of the Two Dimensional Richtmyer-Meshkov Instability}

The primary goal of this study is to examine several numerical methodologies with high order of accuracy for the investigation of two dimensional and eventually three dimensional Richtmyer-Meshkov instability. A detailed description of the evolution of a shock accelerated gaseous interface with different densities under perturbation can be found in paper by Zaytsev et al [1] and references therein. The numerical schemes developed here will served as a basis for the validation of the results obtained from both experiments and other numerical methods. In this preliminary stage, we employ two numerical schemes, namely, high order Pseudospectral methods (Spectral) and Weighted Essentially Non-Oscillatory finite difference scheme (WENO). Detailed discussion of the above mentioned numerical methods can be found in the vast existing literatures.

\section{Governing Equations :}

The governing equations are the two-dimensional Euler equations in Cartesian coordinates describing the conservation of mass, momentum and energy as

$$
\mathrm{Q}_{t}+\mathrm{F}_{x}+\mathrm{G}_{y}=0 .
$$

The vector functions $\mathrm{F}$ and $\mathrm{G}$ are the inviscid fluxes.

The state vector $Q$ is

$$
\mathbf{Q}=(\rho, \rho u, \rho v, E)^{\mathbf{T}},
$$

where $\rho$ is the fluid density, $\mathbf{u}=(u, v)^{\mathrm{r}}$ is the fluid velocity and $E$ is the total energy.

In terms of these variables and the pressure $P=(\gamma-1)\left(E-\rho\left(u^{2}+v^{2}\right)\right)$, the inviscid fluxes $\mathbf{F}$ and $\mathbf{G}$ are given by

$$
\begin{aligned}
& \mathbf{F}=(\rho u, \rho u u+P, \rho u v,(E+P) u)^{\mathrm{r}} \\
& \mathbf{G}=(\rho v, \rho u v, \rho v v+P,(E+P) v)^{\mathrm{r}} .
\end{aligned}
$$

\section{Initial Conditions :}

The physical domain is a rectangular domain with $0 \leq x \leq L_{x}$ and $0 \leq y \leq \lambda$, where $L_{x}$ is the user specified domain length in $x$ and $\lambda$ is the wave length of a single mode perturbation along the interface separating two different gases in the $y$ direction. In this study, the gases are Xenon $(X e)$ and Argon $(A r)$.

The specific heat ratio $\gamma$ is assumed to be the same for both gases, $\gamma=\frac{5}{3}$.

The initial conditions (see figure 1) is an incident shock of Mach number $M=4.46$ located at $x_{s}=0.05 \mathrm{~cm}$ that travels downstrcam toward the interface. Given the shock Mach number $M$, temperature $T$ in the pre-shock region, and the density of the Xenon gas $\rho_{X e}$, the initial condition of the flow satisfies the Hugoniot-Rankine Condition for normal steady shock, i.e., 


$$
\begin{aligned}
T_{2} & =T \\
\rho_{2} & =\rho_{X e} \\
P_{2} & =R \rho_{2} T_{2} \\
C_{2} & =\sqrt{\gamma P_{2} / \rho_{2}} \\
U_{2} & =M C_{2} \\
V_{2} & =0 \\
P_{1} & =P_{2} \frac{2 \gamma M^{2}-(\gamma-1)}{\gamma+1} \\
\rho_{1} & =\rho_{2} \frac{(\gamma+1) M^{2}}{(\gamma-1) M^{2}+2} \\
T_{1} & =P_{1} /\left(R \rho_{1}\right) \\
C_{1} & =\sqrt{\gamma P_{1} / \rho_{1}} \\
U_{1} & =U_{2} \rho_{2} / \rho_{1} \\
V_{1} & =0
\end{aligned}
$$

The subscripts 1 and 2 denote the pre-shock and post-shock condition, respectively. To specify condition for the moving shock, the shock speed $s=M C_{2}$ is subtracted from the pre- and post- shock velocity $U_{1}$ and $U_{2}$, respectively. Using the cgm units, the constant $R=$ $R_{0} / M_{X e}$, where $R_{0}=8.31441 \times 10^{7} \frac{\mathrm{er} g}{K}$ is the universal gas constant, $M_{X e}=131.29 \frac{g}{g-\text { mole }}$ and $M_{A r}=39.948 \frac{g}{g-m o l e}$ are the molecular weight of Xenon and Argon, respectively.

In the pre-shock region, the temperature $T=296.0 \mathrm{~K}$ and the density of Argon and Xenon gases are $\rho_{A r}=0.89 \times 10^{-3} \frac{g}{\mathrm{~cm}^{3}}$ and $\rho_{X e}=2.9 \times 10^{-3} \frac{g}{\mathrm{~cm}^{3}}$ respectively. The pressure is assumed to be half of the normal atmospheric pressure.

Once the pre- and post- shock states of Xcnon gas are specified, the region of Argon gas will be superimposed onto the pre-shock region replacing the Xenon gas. The interface between the two gases is further perturbed to form a sinusoidal wave with some finite thickness, that is, assuming that the interface is initially located at $x_{i}$, for $0 \leq y \leq \lambda$

$$
\begin{array}{ll}
\mathrm{Q}=\mathrm{Q}_{1} & x \leq x_{s} \\
\mathrm{Q}=\mathrm{Q}_{2}+\left(\mathrm{Q}_{\Lambda r}-\mathrm{Q}_{2}\right) S & x>x_{s}
\end{array}
$$

where $\mathrm{Q}$ is the conservative variable, $\mathrm{Q}_{1}$ and $\mathrm{Q}_{2}$ are the post- and pre- shock conditions in the conservative form and $\mathrm{Q}_{A r}$ is the conservative variable of the Argon based on the density of the Argon $\rho_{A r}$ and the same velocity and pressure of the pre-shock condition of the Xenon, namely $U_{2}, V_{2}$ and $P_{2}$ respectively. The mask function $S=S(x, y)$ is defined as

$$
\begin{array}{lcl}
S= & 1 & d \leq 0 \\
S= & \exp \left(-\alpha|d|^{\beta}\right) & 0<d<1 \\
S= & 0 & d \geq 1
\end{array}
$$


where

$$
d=\frac{\left(x_{i}+a \cos (2 \pi y / \lambda)+\delta\right)-x}{2 \delta}
$$

and $\delta>0$ and $\beta>0$ are the interface thickness and its order respectively, $\alpha=-\ln \epsilon$ with $\epsilon$ being the machine zero, $a=1.0 \mathrm{~cm}$ and $\lambda=3.6 \mathrm{~cm}$ are the amplitude and wave length of the perturbation.

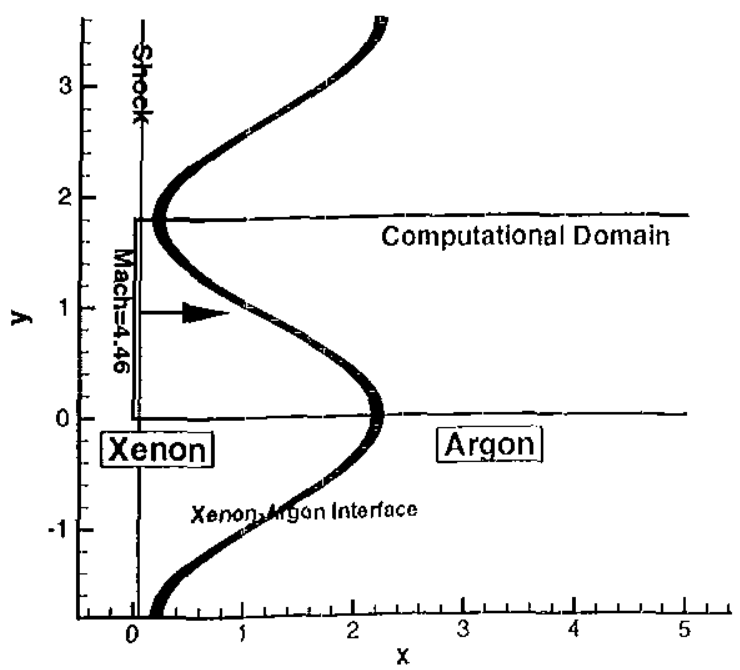

Figure 1: Initial Condition of the Richtmyer-Meshkov instability problem.

\section{Solution Procedure :}

1. Periodical Domain is specified in the $y$ direction and symmetry property of the problem is exploited to reduce the amount of computational operations by half.

2. A combined Chebyshev and Fourier collocation methods is used to discretize the Euler equations in space yiclding a Spectral scheme. A WENO-LF-5 fifth order finite difference scheme is also employed for the solution of the problem.

3. The software library PseudoPack, written by Bruno Costa and Wai Sun Don, is utilized for the Spectral scheme.

4. The third order TVD Runge Kutta method by Shu and Osher is used to advance the solution in time.

5. For the Spectral scheme, a 10'th and 9'th order exponential filter is used for the differentiation and solution smoothing respectively, at each Runge Kutta step unless otherwise specified.

6. The domain length $L_{x}$ is specified sufficiently large to retain the transmitted shock within the physical domain. 
7. $\beta=8$ is used for the definition of the interface thickness order. The interface thickness $\delta$ is specified by user depending on how diffuse the interface is and the resolution power of the numerical scheme.

\section{Results :}

A scries of numerical simulations are carried out to investigate the convergence properties of both the Spectral scheme and the WENO scheme. Simulations, using various interface thicknesses and resolutions, are computed and terminated at some representative time after the shock had transmitted sufficiently far away from the interface and before exiting the physical domain. It allows the development of vortical rollups of the gaseous interface. Vorticities are generated by the cross product of the pressure gradient of the shock and the density gradient of the gases. The final time is set to $t=50 \times 10^{-6} \mathrm{~s}$ for $L_{x}=50 \mathrm{~cm}$ and $t=143 \times 10^{-6} \mathrm{~s}$ for $L_{x}=150 \mathrm{~cm}$.

As evidenced from the results of the Spectral and the WENO calculations shown below, the following major features of the Richtmyer-Meshkov instability can be observed (see figure 2) at time $t=50 \times 10^{-6} \mathrm{~s}$, namely,

- Wave generated by the shock refraction behind the gas interface in Box 1 .

- The penetration of the heavy (Xc) to light (Ar) fluid causes the deformation of the interface into a large mushroom shape structures in Box 2 and the opposite in Box 5. They are refered as Spike and Bubble respectively, in the literatures. They move in the opposite direction relative to each other and form a ever larger turbulence mixming zone.

- Pressure wave along the transmitted shock in Box 3.

- A small jet and its vortical structure located in Box 4. The contact discontinuity develops into a more complicated vortical rollups in a finer and long term simulation (see figure 17) possibly caused by the Kelvin-Helmholtz instability.

- Vortical rollups of the gascous interface inside Box 6.

The global large and median features (Box 1, 2, 3, 4 and 5) are well captured accurately by both numerical schemes for a given resolution. It is unclear, however, if the smaller rollups along the gases interface (Box 6) presented in the high resolution/high order cases are physical due to the non-dissipative nature of the Euler equations or numerical due to the oscillatory nature of the numerical schemes or both. More researches are needed to answer this question fully.

For long time integration, the smoothest of the gaseous interface at the earlier development yields a slightly smoother and rounder interface shape at the later time as seen in the WENO calculation. The overall global structures, however, seem to agree very well among the calculations performed here. (see figures (17-18)).

Convergence Study : $\delta=0.6 \mathrm{~cm}$ 


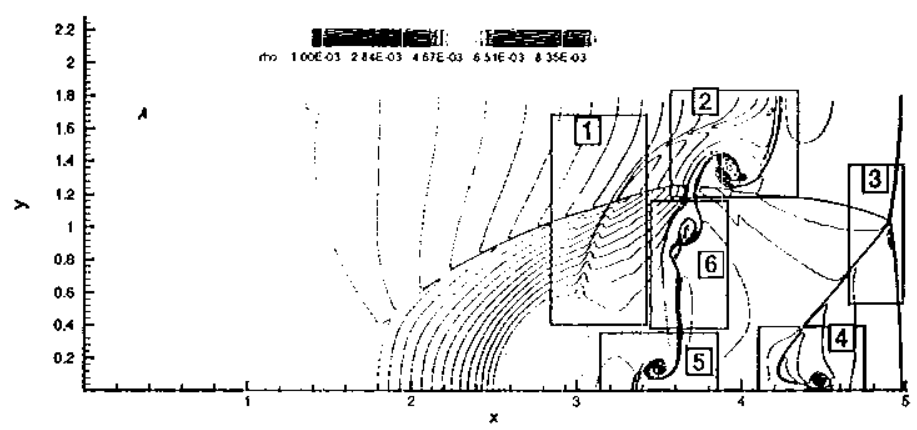

Figure 2: The numbered regions enclose the most prominent flow features of the RichmyterMeshkov instability at time $t=50 \times 10^{-6} \mathrm{~s}$.

We shall first examine the convergence property of both the Spectral scheme and the WENO-LF-5 finite difference scheme. For this, we used a thicker interface with $\delta=0.6 \mathrm{~cm}$ to establish the convergence of the numerical schemes of the large and medium scale structures (box 1, 2, 3, 4 and 5 in figure 2). This avoids the possible contamination of numerical artifacts due to high gradients generated along the shock-interface interaction and bypass the issue of under-resolved fine scale physical structures.

Figures $(3-6)$ show the density $\rho$, velocity $U$ and $V$ and total energy $E$ of the solution of the Spectral and WENO-LF-5 scheme at time $t=50 \times 10^{-6} \mathrm{~s}$ at various resolutions. It can observed that the large and medium scale structures such as the transmitted shock, the location of the triple point, the shocked-interface velocity, pressure waves and vorticity generation, are basically in excellent agreement with each other.

Convergence Study : $\delta=0.2 \mathrm{~cm}$

The density $\rho$, velocity $U$ and $V$ and total energy $E$ of the solution of the Spectral scheme (figures $(7-10)$ ) and WENO-JF-5 scheme (figures $(12-15)$ ) at time $t=50 \times 10^{-6} \mathrm{~s}$ at various resolutions. The interface thickness is significantly reduced from $\delta=0.6 \mathrm{~cm}$ to $\delta=0.2 \mathrm{~cm}$. An eighth order exponential filter is used for the Spectral scheme with resolution of $384 \times 192$.

Similar to the previous case of $\delta=0.6 \mathrm{~cm}$, it can be observed that the large and median scale structures such as transmitted shock, shocked-interface velocity and shock triple point are basically in excellent agreement with each others. Some discrepancies of the fine scale structures along the gaseous interface, as can be expected for numerical simulation of the Euler equations which is sensitive to perturbation in nature, are observed.

Snapshot of the evolution of density and velocity flow fields at several immediate times are illustrated in figure (11), for the Spectral scheme and in figure (11) for the WENO scheme. The contour levels are the same and constant for both schemes in all plots.

The Mach number $M$, the Atwood number $A t$ and the interface curvature play an important role on the growth of perturbed amplitude on the interface. In the particular set of parameters studied here with high Mach number $M=4.46$ and median Atwood number $A t \approx 5.4$, a formation of triple-shock configuration along the interface indicates that shockinterface interaction is in the "hard" regime. A "hard" regime, as quoted from Zaytsev etc. 
is "the propagation of secondary shocks across the flow that is accompanied by the formation of breaks and triple configurations on the refracted and reflected shocks". The triple-shock formation can be observed easily in the carly time $<\approx 30 \times 10^{-6} s$.

\section{Large Domain/Long time Study :}

To observe the long term cvolution of the interface, the domain in $x$ is increased from $L_{x}=5 \mathrm{~cm}$ to $L_{x}=15 \mathrm{~cm}$ and the final time is increased from $t=50 \times 10^{-6} \mathrm{~s}$ to $t=$ $143 \times 10^{-6} s$, see figures (17-18). Other than the slightly smoother solutions computed by the WENO scheme and fine scale structures along the gaseous interface, the large and medium scale structures of the flow ficlds are well captured by both numerical schemes.

The amplitude of the perturbed interface $a$ is first decreased by the compression of the shock wave. The interface is then accelerated by the shock and grows from $2 \mathrm{~cm}$ to $\approx 2.6 \mathrm{~cm}$ at time $143 \times 10^{-6} \mathrm{~s}$. It seems to match the experimental data given in figure 1.9 in the paper by Zaytsev et al. for the case of initial amplitude $a_{0}=1 \mathrm{~cm}$ and the distance passed by shock after interaction with the interface $X_{K_{12}} \approx 13 \mathrm{~cm}$.

\section{Research :}

In the next phase of the rescarch, we will study the single or combined effects of the Mach number $M$, the amplitude $a$ and wavelength $\lambda$ of the interface, different gas composition $\rho$, longer time bchavior of the interface evolution and validation of the results with the available experimental data. We might also consider replacing the Euler equations with the multi-species Navier-Stokes equations that corresponds better with the nature of the Richtmyer-Meshkov instability in experiments.

\section{References}

[1] A. N. Aleshin, E. I. Chebotareva, V. V. Krivets, E. V. Lazareva, S. V. Sergeev, S.N. Titov and S. G. Zaytsev Investigation of Evolution of Interface After Its Interaction with Shock Waves, International Institute for Applied Physics and High Technology, Report No. 06-96 

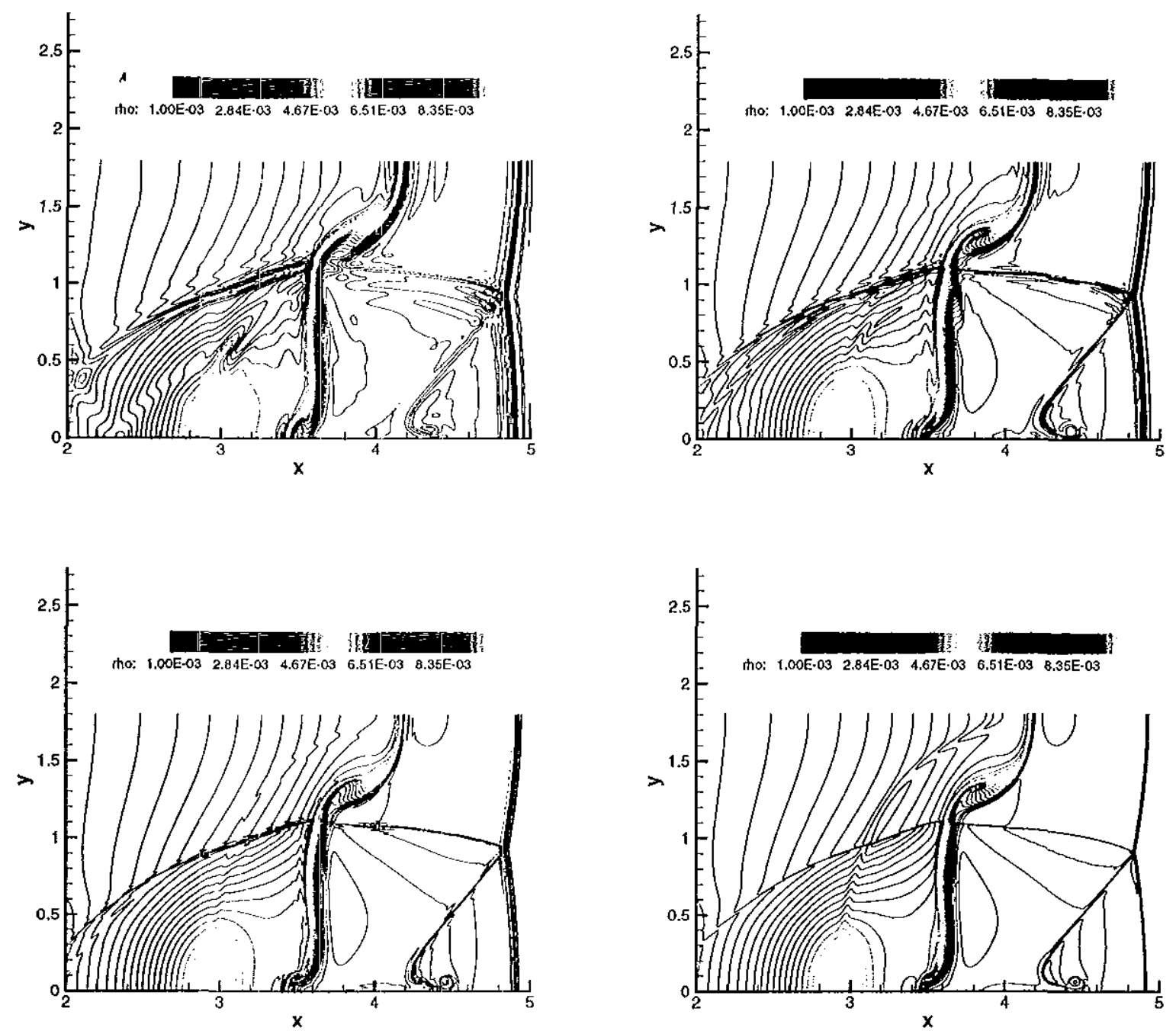

Figure 3: Convergence Study $\delta=0.6 \mathrm{~cm}$ : Density contour plot of the Richtmyer-Meshkov instability as computed by the Spectral scheme and the WENO-LF-5 scheme. Domain length in $x$ is $L_{x}=5 \mathrm{~cm}$. The interface thickness $\delta=0.6 \mathrm{~cm}$. The final time is $t=50 \times 10^{-6} \mathrm{~s}$.

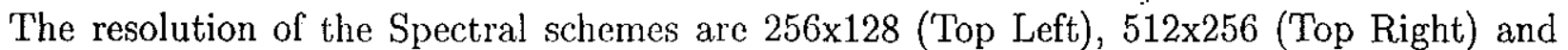
$1024 \times 512$ (Bottom Left) and the WENO scheme is $1024 \times 512$ (Bottom Right). 

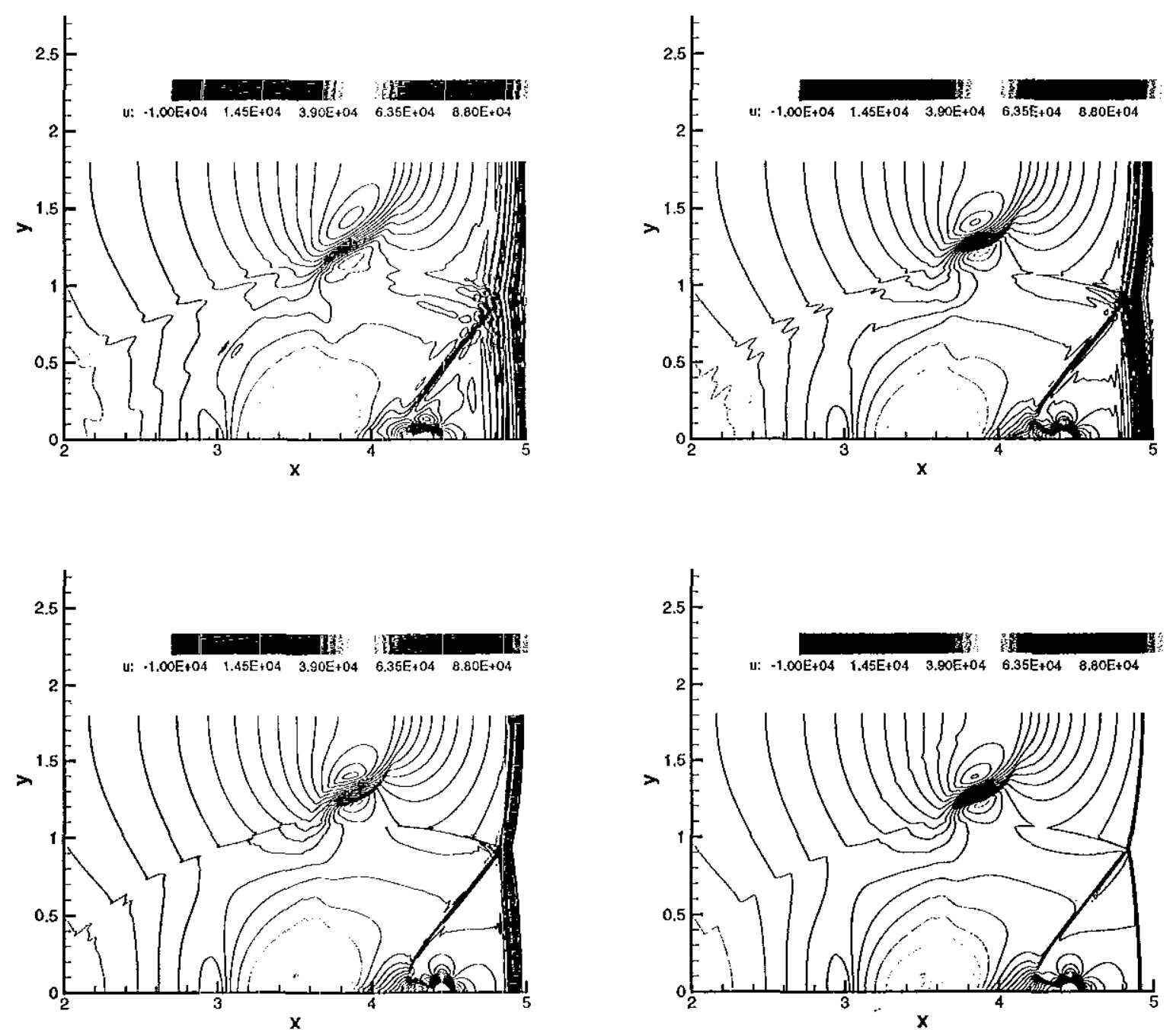

Figure 4: Convergence Study $\delta=0.6 \mathrm{~cm}$ : U-Velocity contour plot of the Richtmyer-Meshkov instability as computed by the Spectral scheme and the WENO-LF-5 scheme. Domain length in $x$ is $L_{x}=5 \mathrm{~cm}$. The interface thickness $\delta=0.6 \mathrm{~cm}$. The final time is $t=50 \times 10^{-6} \mathrm{~s}$. The resolution of the Spcctral schemes are 256x128 (Top Left), 512x256 (Top Right) and $1024 \times 512$ (Bottom Left) and the WENO scheme is 1024x512 (Bottom Right). 

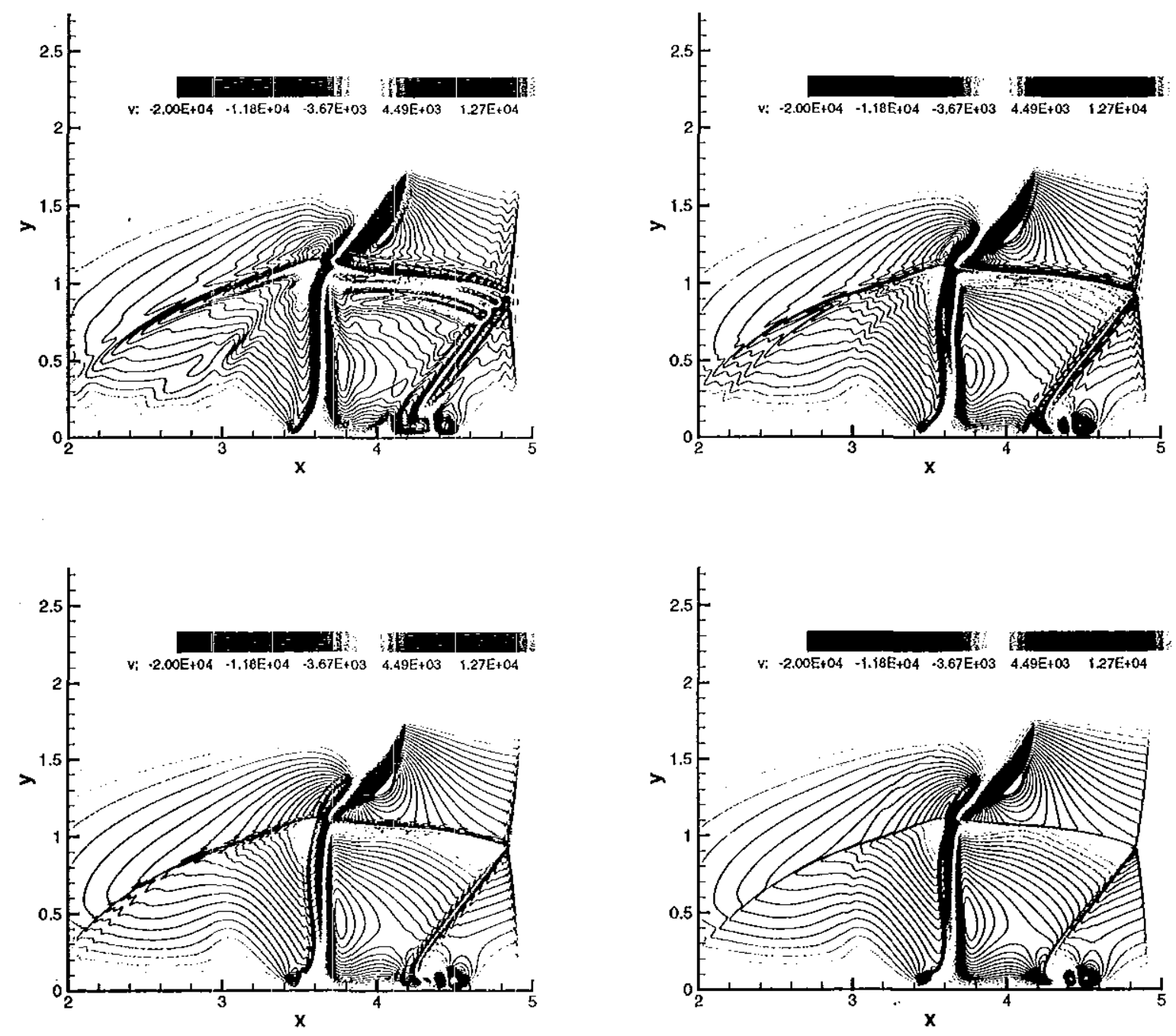

Figure 5: Convergence Study $\delta=0.6 \mathrm{~cm}:$ V-Velocity contour plot of the Richtmyer-Meshkov instability as computed by the Spectral scheme and the WENO-LF-5 scheme. Domain length in $x$ is $L_{x}=5 \mathrm{~cm}$. The interface thickness $\delta=0.6 \mathrm{~cm}$. The final time is $t=50 \times 10^{-6} \mathrm{~s}$.

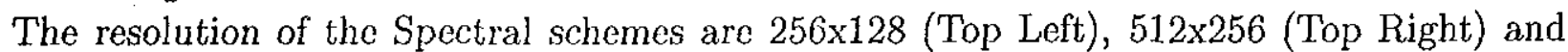
$1024 \times 512$ (Bottom Left) and the WENO scheme is 1024x512 (Bottom Right). 

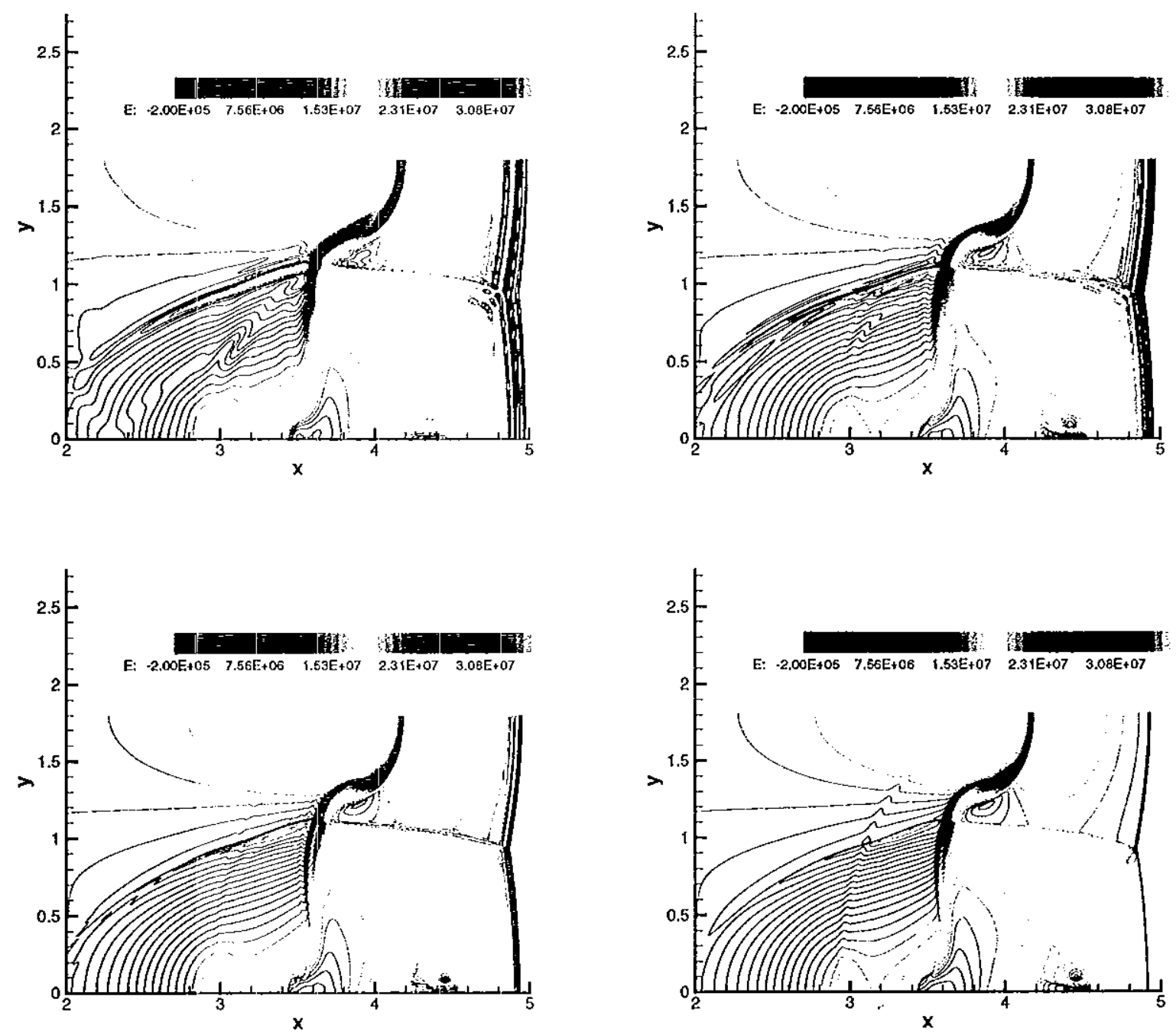

Figure 6: Convergence Study $\delta=0.6 \mathrm{~cm}$ : Total Energy contour plot of the RichtmyerMeshkov instability as computed by the Spectral scheme and the WENO-LF-5 scheme. Domain length in $x$ is $L_{x}=5 \mathrm{~cm}$. The interface thickness $\delta=0.6 \mathrm{~cm}$. The final time is

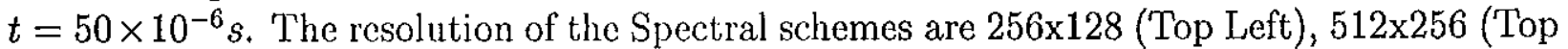
Right) and 1024x512 (Bottom Left) and the WENO scheme is 1024x512 (Bottom Right). 

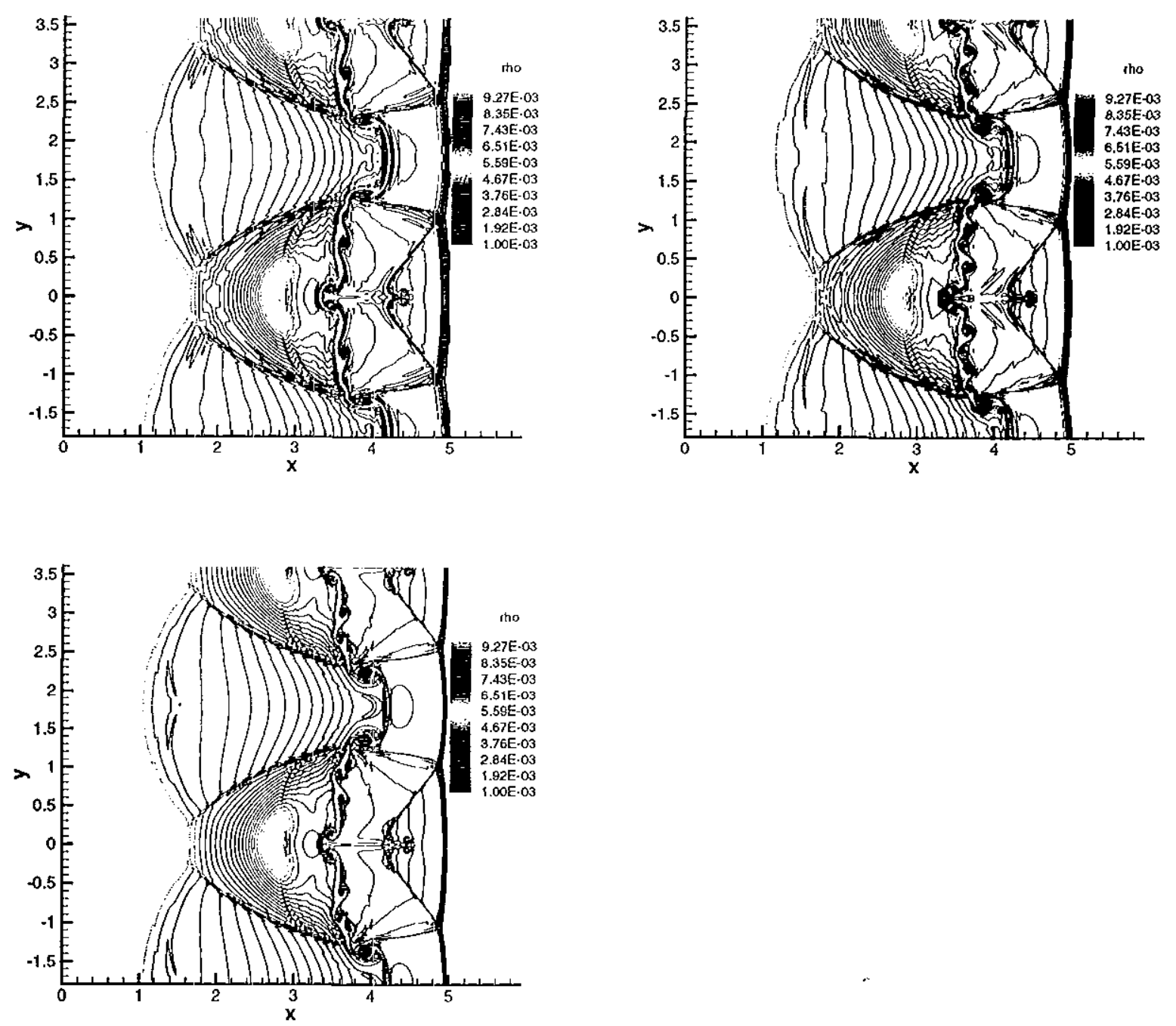

Figure 7: Convergence Study $\delta=0.2 \mathrm{~cm}$ : Density contour plot of the Richtmyer-Meshkov instability as computed by the Spectral scheme. Domain length in $x$ is $L_{x}=5 \mathrm{~cm}$. The interface thickness $\delta=0.2 \mathrm{~cm}$. The final time is $t=50 \times 10^{-6} \mathrm{~s}$. The resolution of the Spectral schemes are 384x192 (Top Left), 512x256 (Top Right) and 1024x256 (Bottom Left). 

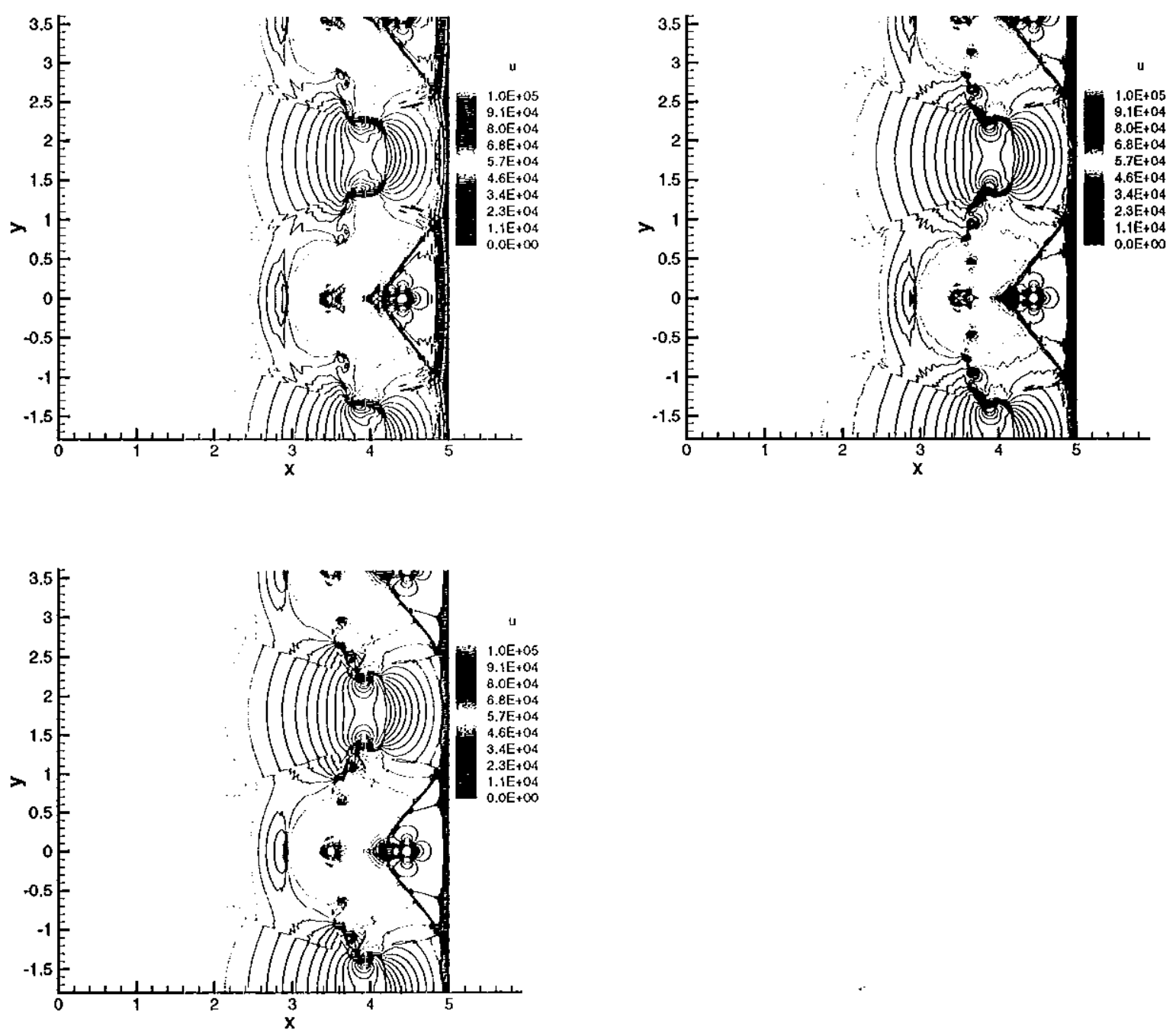

Figure 8: Convergence Study $\delta=0.2 \mathrm{~cm}$ : U-Velocity contour plot of the RichtmyerMeshkov instability as computed by the Spectral scheme. Domain length in $x$ is $L_{x}=5 \mathrm{~cm}$. The interface thickness $\delta=0.2 \mathrm{~cm}$. The final time is $t=50 \times 10^{-6} \mathrm{~s}$. The resolution of the Spectral schemes are 384x192 (Top Left), 512x256 (Top Right) and 1024x256 (Bottom Left). 

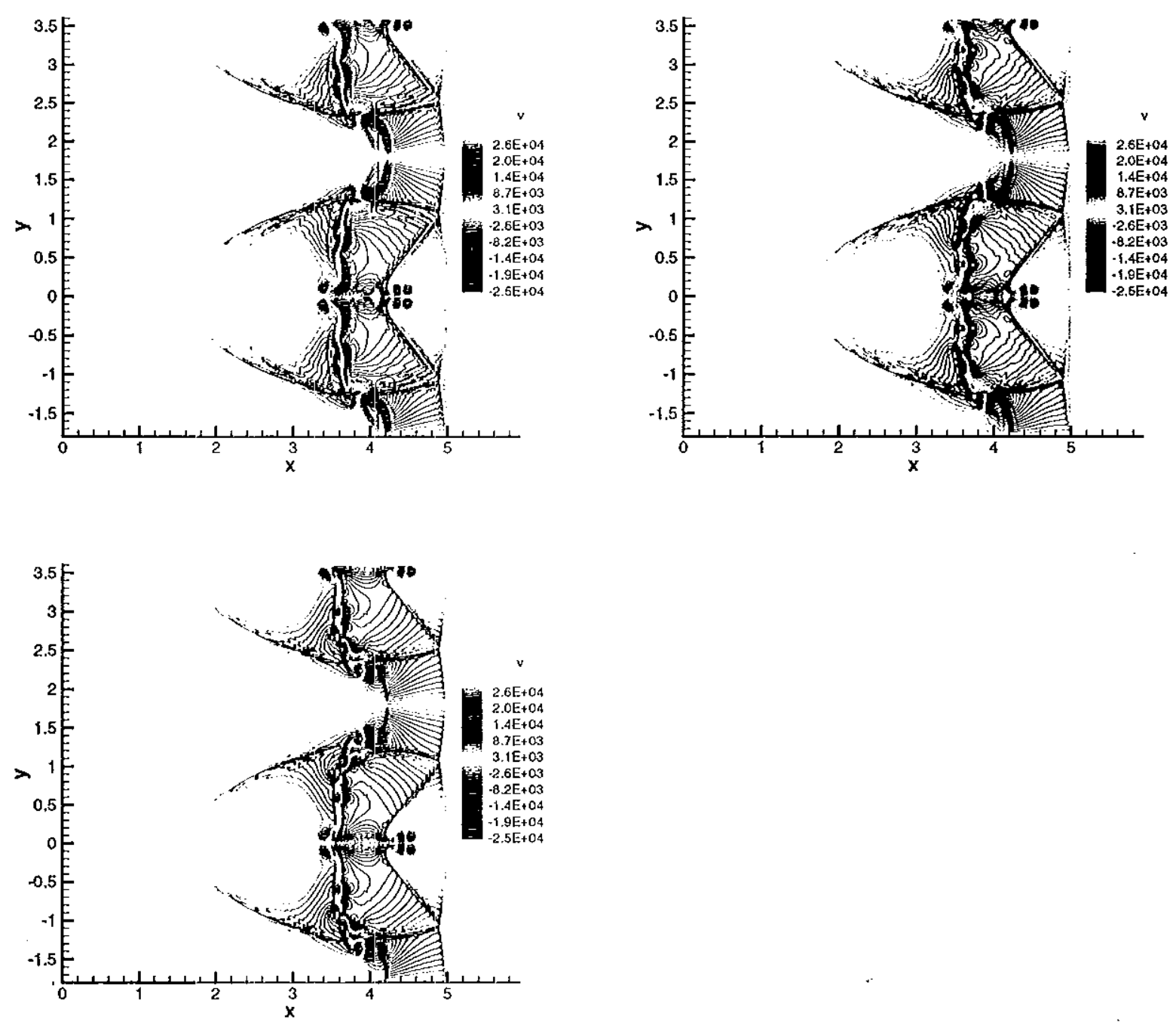

Figure 9: Convergence Study $\delta=0.2 \mathrm{~cm}$ : V-Velocity contour plot of the RichtmyerMeshkov instability as computed by the Spectral scheme. Domain length in $x$ is $L_{x}=5 \mathrm{~cm}$. The interface thickness $\delta=0.2 \mathrm{~cm}$. The final time is $t=50 \times 10^{-6} \mathrm{~s}$. The resolution of the Spectral schemes are 384x192 (Top Left), 512×256 (Top Right) and 1024x256 (Bottom Left). 

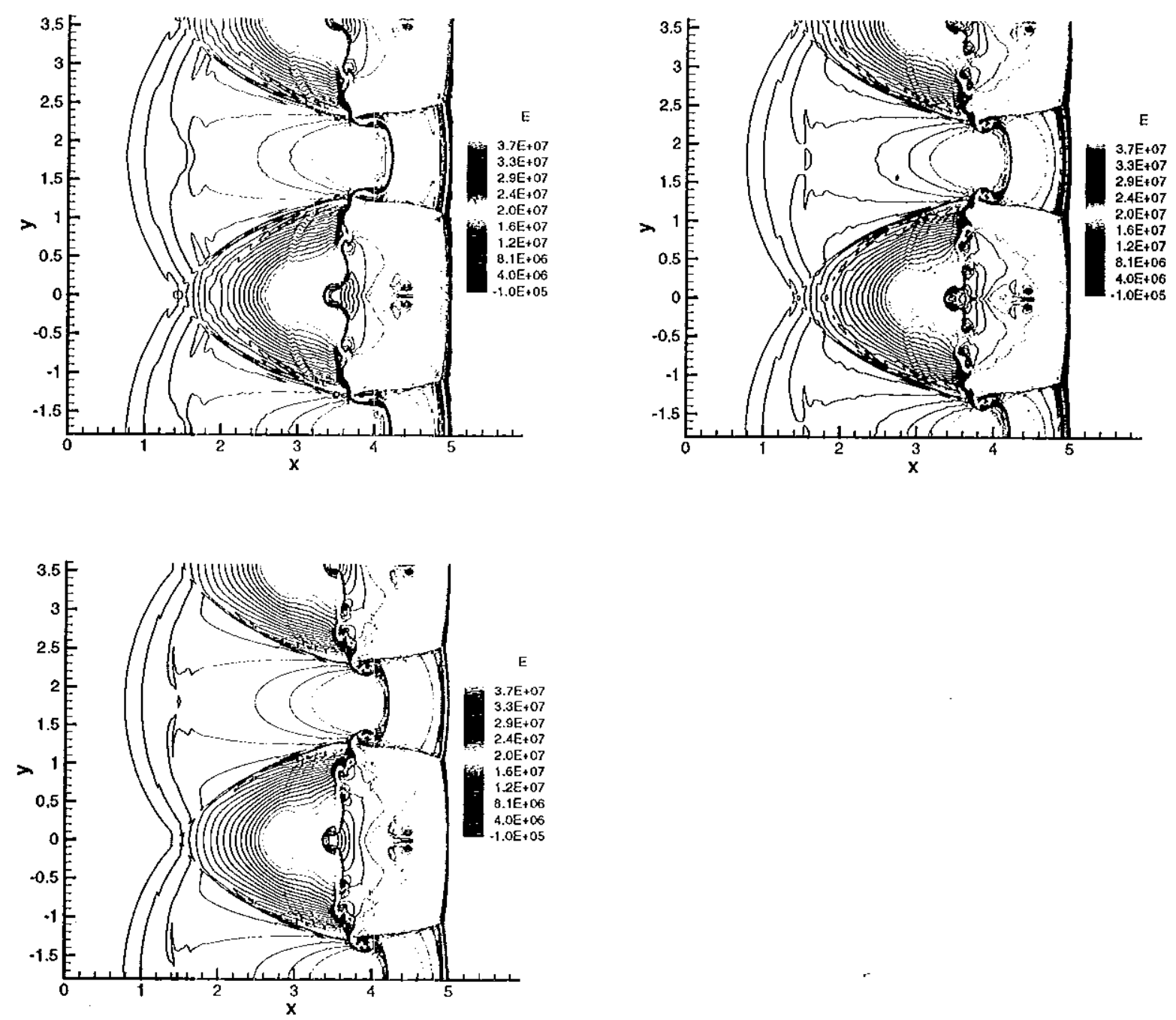

Figure 10: Convergence Study $\delta=0.2 \mathrm{~cm}$ : Total Energy contour plot of the RichtmyerMeshkov instability as computed by the Spectral scheme. Domain length in $x$ is $L_{x}=5 \mathrm{~cm}$. The interface thickness $\delta=0.2 \mathrm{~cm}$. The final time is $t=50 \times 10^{-6} \mathrm{~s}$. The resolution of the Spectral schemes are 384x192 (Top Left), 512x256 (Top Right) and 1024x256 (Bottom Left). 


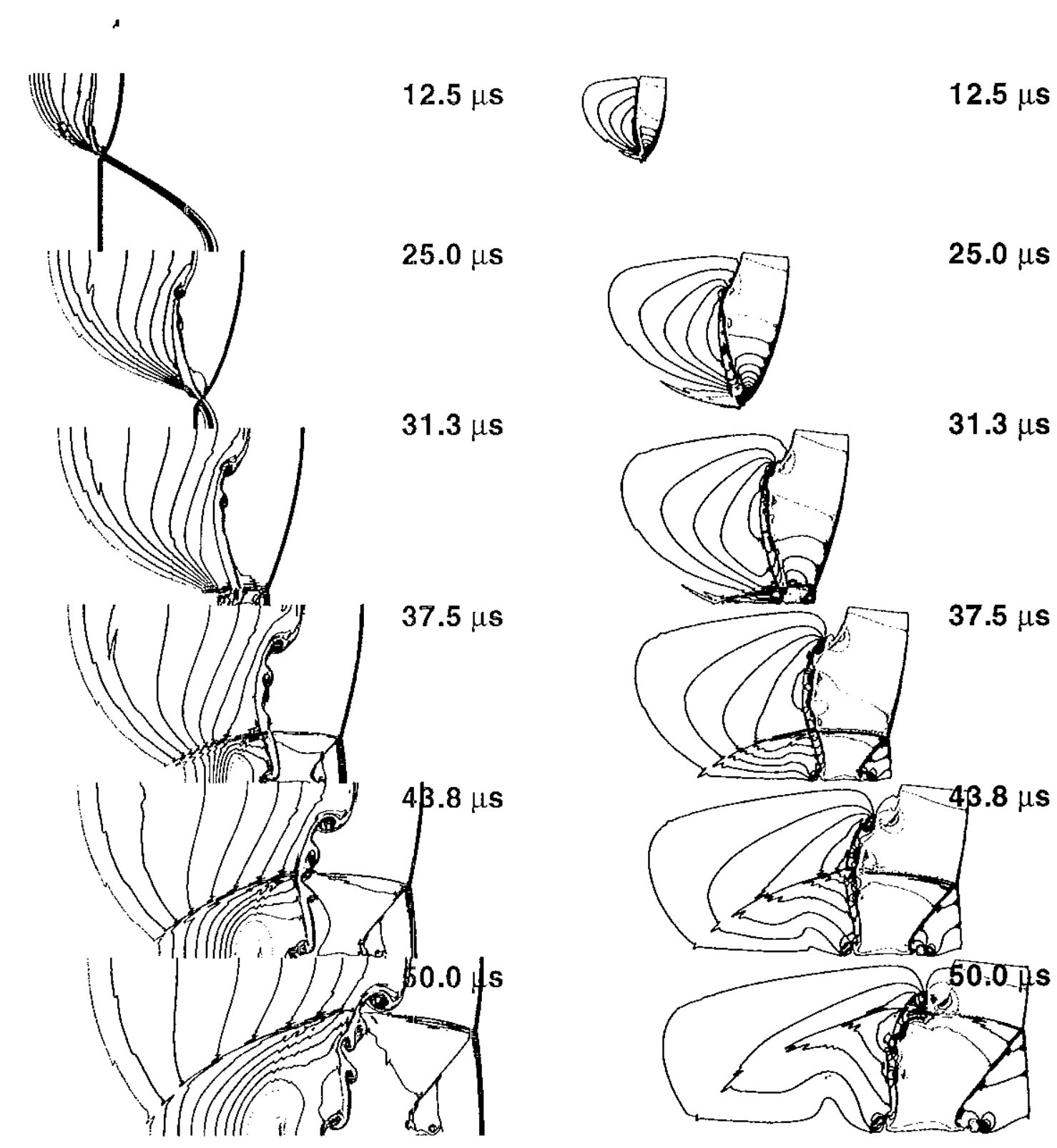

Figure 11: Convergence Study $\delta=0.2 \mathrm{~cm}$ : Density (Left) and Velocity (Right) contour plot of the Richtmyer-Meshkov instability as computed by the Spectral scheme at time $t=12.5 \times 10^{-6} s, 25.0 \times 10^{-6} s, 31.3 \times 10^{-6} s, 37.5 \times 10^{-6} s, 43.8 \times 10^{-6} s$ and $50.0 \times 10^{-6} s$. Domain length in $x$ is $L_{x}=5 \mathrm{~cm}$. The interface thickness $\delta=0.2 \mathrm{~cm}$. The resolution of the Spectral scheme is $1024 \times 256$. 

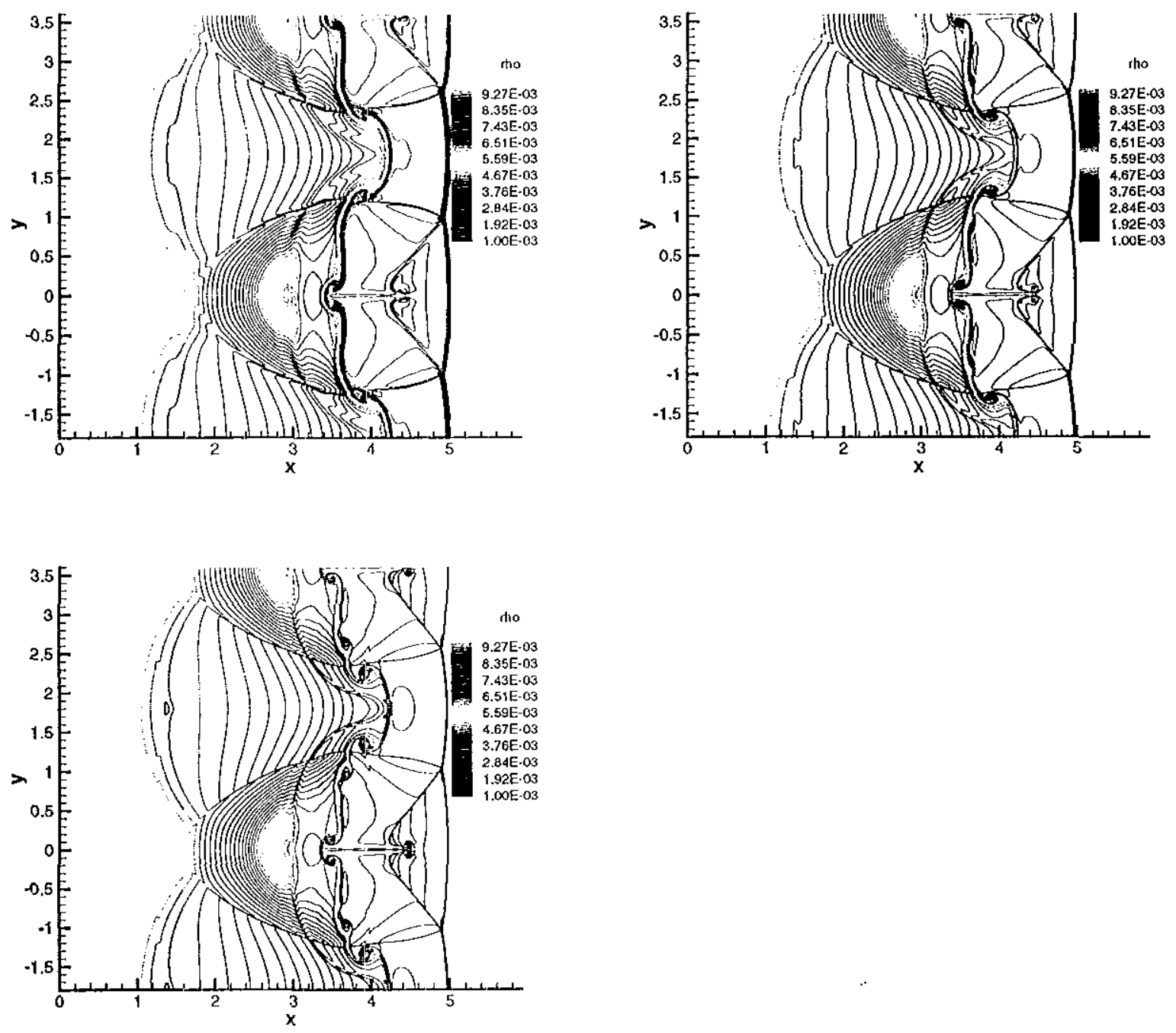

Figure 12: Convergence Study $\delta=0.2 \mathrm{~cm}$ : Density contour plot of the Richtmyer-Meshkov instability as computed by the WENO-JF-5scheme. The final time is $t=50 \times 10^{-6} \mathrm{~s}$. The resolution of the WENO-LF-5 schemes are 256x128 (Top Left), 512×256 (Top Right) and 1024x512 (Bottom Left). 

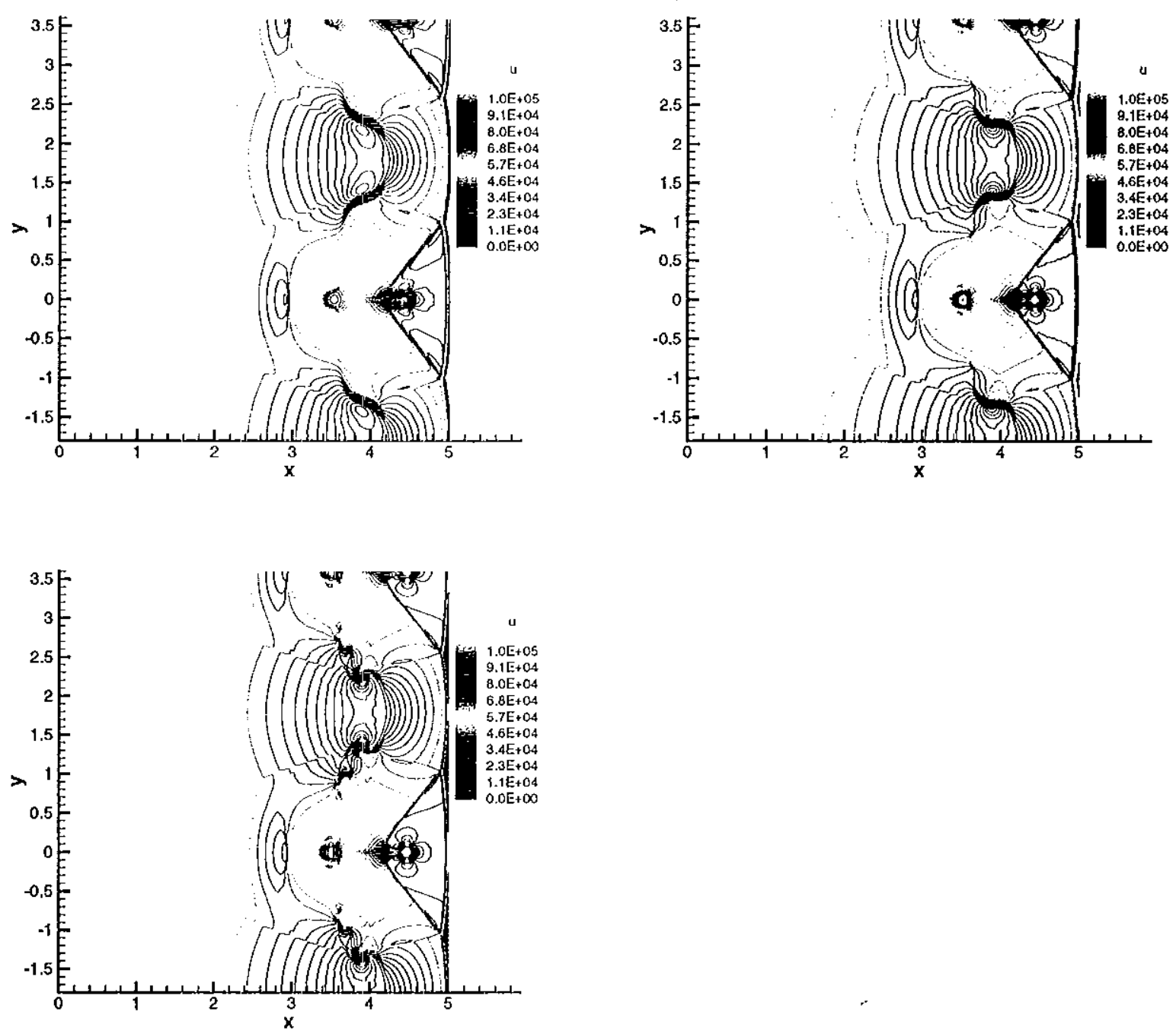

Figure 13: Convergence Study $\delta=0.2 \mathrm{~cm}$ : U-Velocity contour plot of the RichtmyerMeshkov instability as computed by the WENO-LF-5scheme. Domain length in $x$ is $L_{x}=$ $5 \mathrm{~cm}$. The interface thickness $\delta=0.2 \mathrm{~cm}$. The final time is $t=50 \times 10^{-6} \mathrm{~s}$. The resolution of the WENO-LF-5 schemes are 256x128 (Top Left), 512x256 (Top Right) and 1024x512 (Bottom Left). 

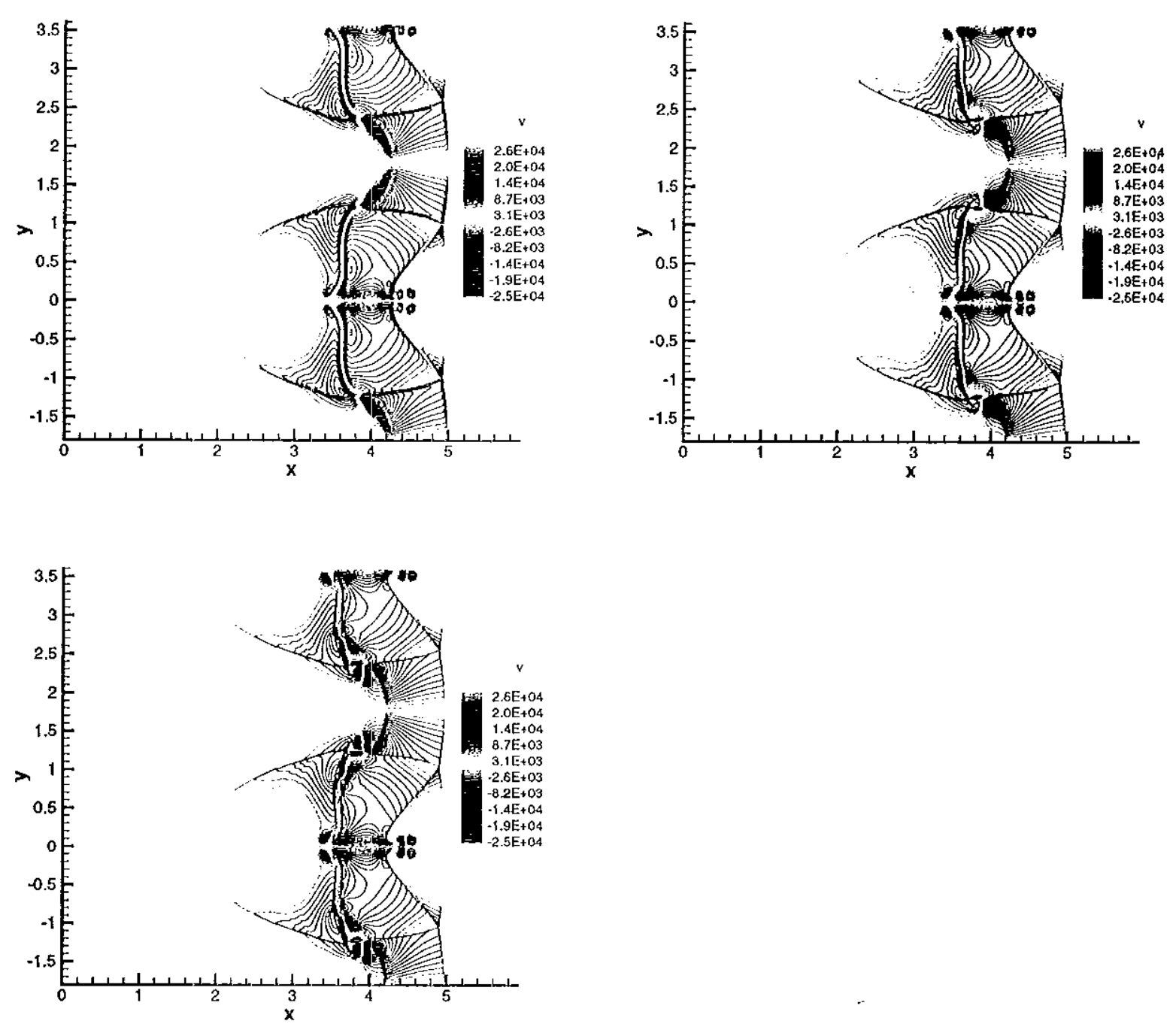

Figure 14: Convergence Study $\delta=0.2 \mathrm{~cm}$ : V-Velocity contour plot of the RichtmyerMeshkov instability as computed by the WENO-LF-5scheme. Domain length in $x$ is $L_{x}=$ $5 \mathrm{~cm}$. The interface thickness $\delta=0.2 \mathrm{~cm}$. The final time is $t=50 \times 10^{-6} \mathrm{~s}$. The resolution

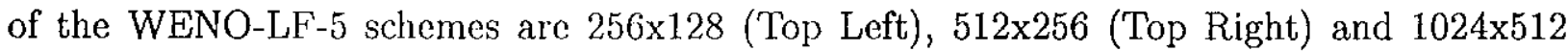
(Bottom Left). 

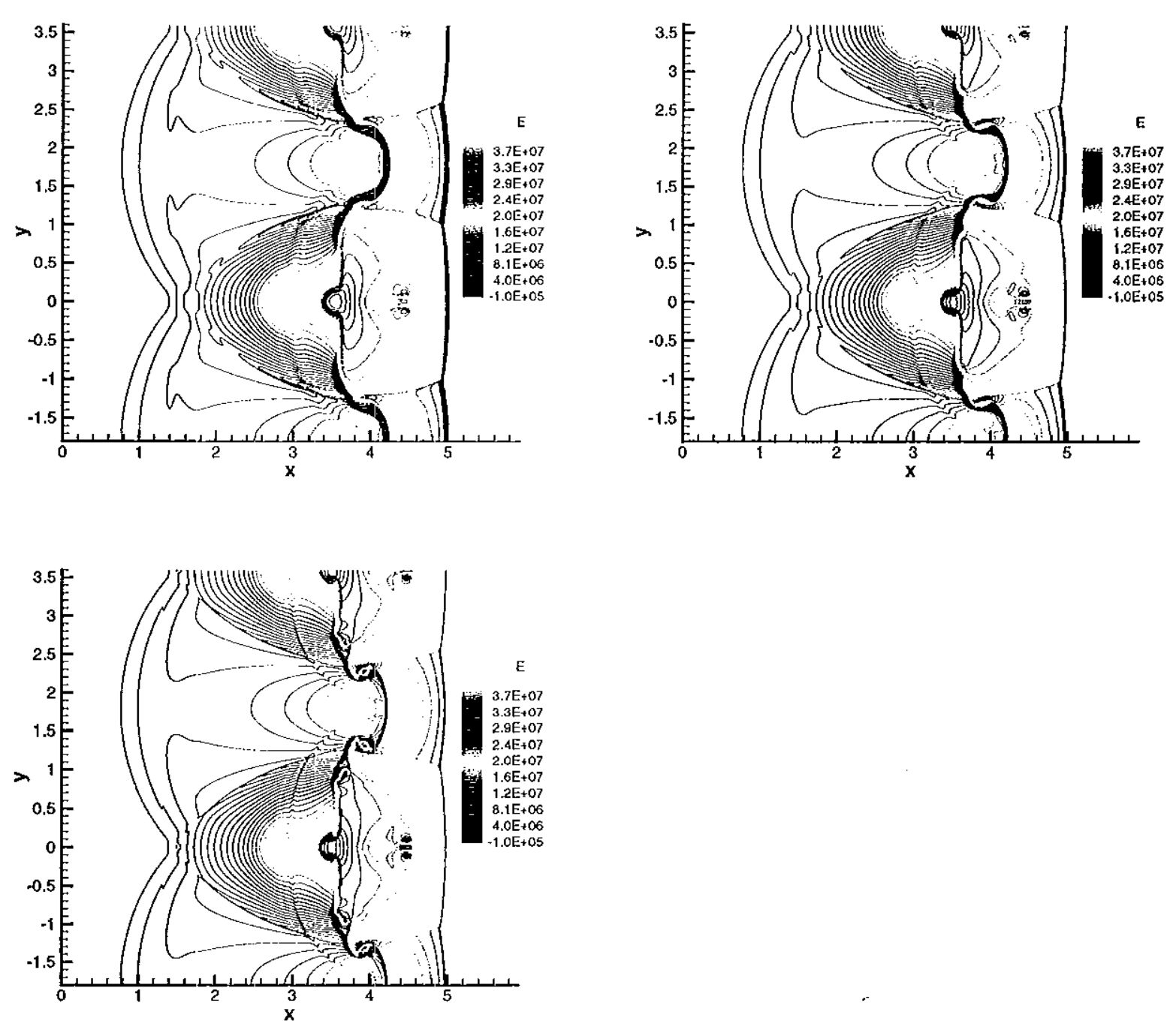

Figure 15: Convergence Study $\delta=0.2 \mathrm{~cm}$ : Total Energy contour plot of the RichtmyerMeshkov instability as computed by the WENO-LF-5scheme. Domain length in $x$ is $L_{x}=$ $5 \mathrm{~cm}$. The interface thickness $\delta=0.2 \mathrm{~cm}$. The final time is $t=50 \times 10^{-6} \mathrm{~s}$. The resolution of the WENO-LF-5 schemes are 256x128 (Top Left), 512x256 (Top Right) and 1024x512 (Bottom Left). 


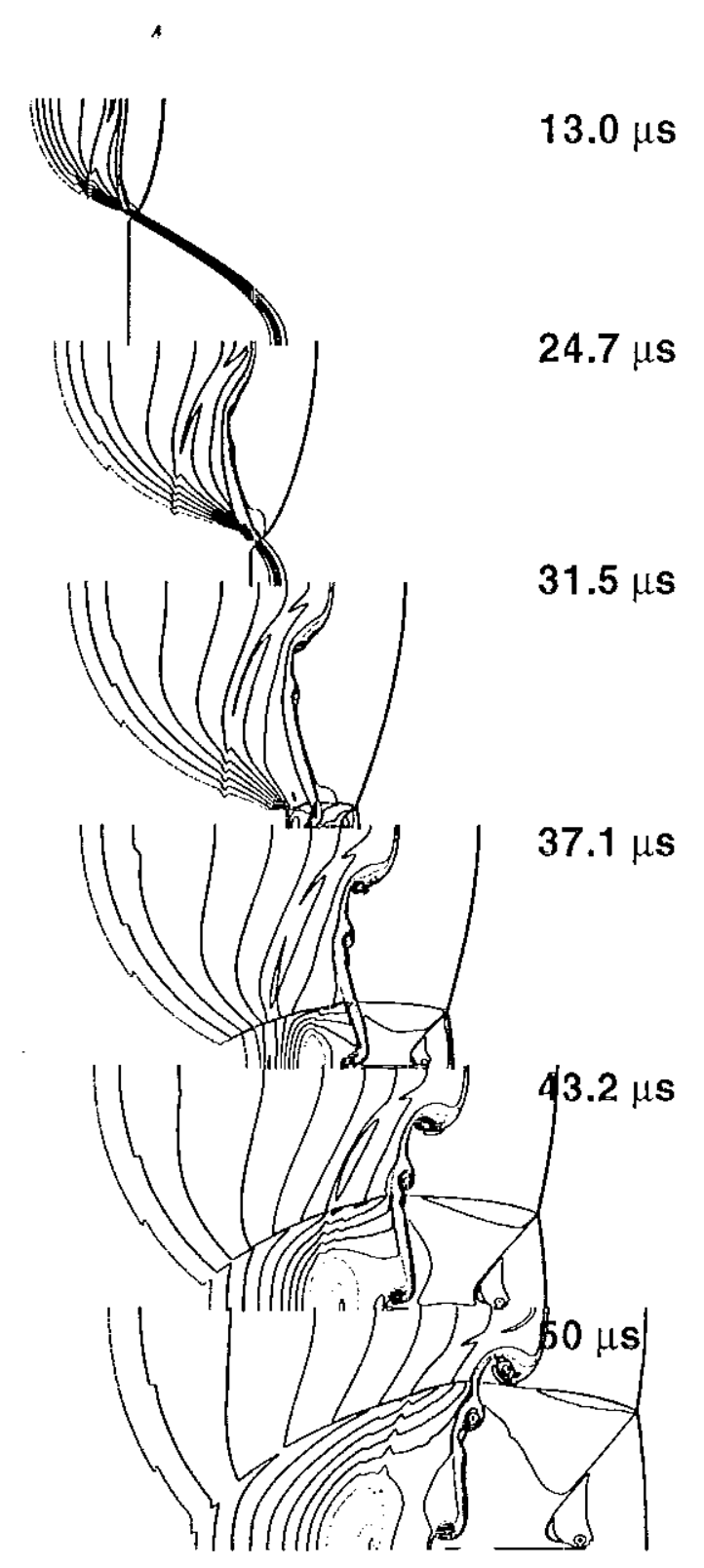

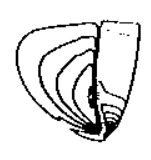
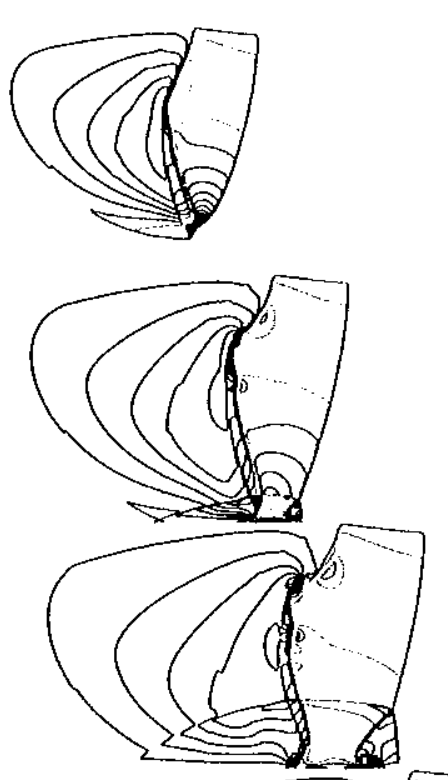

$31.5 \mu \mathrm{s}$

$37.1 \mu \mathrm{s}$

Figure 16: Convergence Study $\delta=0.2 \mathrm{~cm}$ : Density (Left) and Velocity (Right) contour plot of the Richtmyer-Meshkov instability as computed by the WENO-LF-5 scheme at time $t=13.0 \times 10^{-6} s, 24.7 \times 10^{-6} s, 31.5 \times 10^{-6} s, 37.1 \times 10^{-6} s, 43.2 \times 10^{-6} s$ and $50.0 \times 10^{-6} s$. Domain length in $x$ is $L_{x}=5 \mathrm{~cm}$. The interface thickness $\delta=0.2 \mathrm{~cm}$. The resolution of the WENO-LF-5 scheme is $1024 \times 512$. 

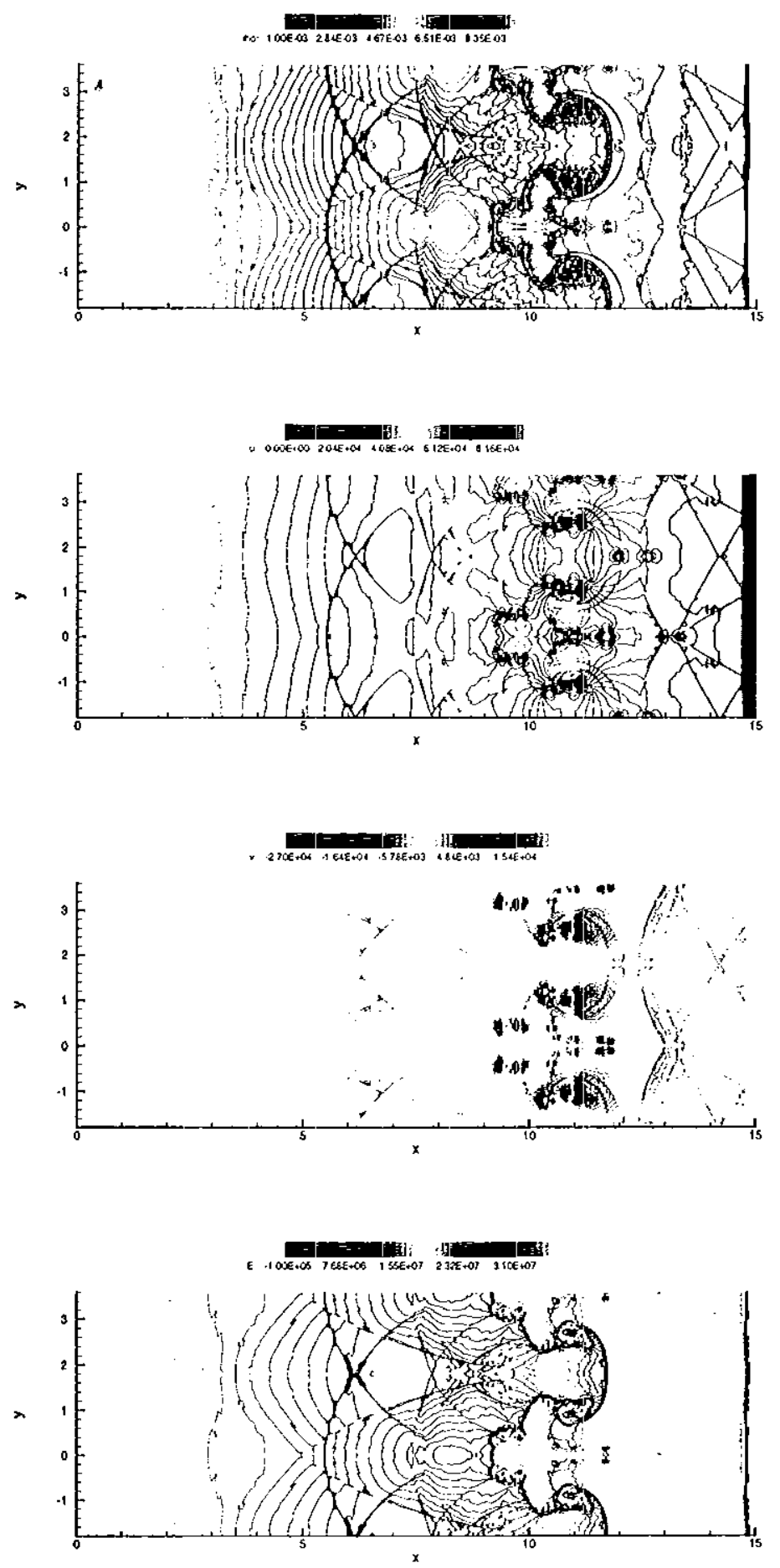

Figure 17: Large Domain Study : Density (Top Left), U-Velocity (Top Right), V-Velocity (Bottom Left) and Total Energy (Bottom Right) contour plot of the Richtmyer-Meshkov instability as computed by the Spectral scheme. Domain length in $x$ is $L_{x}=15 \mathrm{~cm}$. The interface thickness $\delta=0.2 \mathrm{~cm}$. The final time is $t=143 \times 10^{-6} \mathrm{~s}$. The resolution of the spectral schemes is $1536 \times 512$. 